\title{
Development and Evaluation of Median Lethal Concentration (LC50) of Wettable Powder and Oil Based Formulations of Lecanicillium lecanii (Zimmermann) IOF1 Strain (KM215209) under in vitro Conditions
}

\author{
Sharanabasappa M. Ganganalli* and R.K. Patil \\ Department of Agricultural Entomology, University of Agricultural Sciences, \\ Dharwad, India
}

*Corresponding author

\begin{abstract}
A B S T R A C T
Keywords

The present in vitro studies on bio-efficacy of granular, oil based and wettable powder formulations on various sucking pests were carried out at Entomology laboratory, Institute

Lecanicillium

lecanii, LC50,

Formulation

of Organic Farming (IOF), University of Agricultural Sciences, Dharwad. Among different formulations evaluated viz., rice bran oil $(60 \%)+$ corn oil $(40 \%)$ formulation found least $\mathrm{LC}_{50}$ value against corn aphids $\left(0.182 \times 10^{6} \mathrm{cfu} / \mathrm{ml}\right)$, grape vine mealy bug $\left(0.560 \times 10^{6}\right.$ $\mathrm{cfu} / \mathrm{ml})$, cotton thrips $\left(0.591 \times 10^{6} \mathrm{cfu} / \mathrm{ml}\right)$, and guava whitefly $\left(0.942 \times 10^{6} \mathrm{cfu} / \mathrm{ml}\right)$. The olive oil formulation recorded least $\mathrm{LC}_{50}$ value $0.674 \times 10^{6} \mathrm{cfu} / \mathrm{ml}$ was against

Article Info

Accepted:

12 January 2019

Available Online:

10 February 2019 soybean mite. The wettable powder formulation found inferior by recording highest $\mathrm{LC}_{50}$ value against corn aphid $\left(0.261 \times 10^{8} \mathrm{cfu} / \mathrm{g}\right)$, grape vine mealybug $\left(0.740 \times 10^{8} \mathrm{cfu} / \mathrm{g}\right)$, cotton thrips $\left(1.019 \times 10^{8} \mathrm{cfu} / \mathrm{g}\right)$, guava whitefly $\left(1.757 \times 10^{8} \mathrm{cfu} / \mathrm{g}\right)$ and soybean mite $\left(0.917 \times 10^{8} \mathrm{cfu} / \mathrm{g}\right)$ at $120 \mathrm{~h}$. Oil formulations are compatible with other integrated pest management approaches. These formulations provide scope for the application of entomopathogens in arid climate where the temperature and relative humidity are major constraints.
\end{abstract}

\section{Introduction}

In recent past, increased environmental awareness, failure of conventional chemical insecticides and pesticides, increased number of insecticide resistant species and food safety and concerns, the application of biological control is amplifying abundantly (Digvijay Singh et al., 2017). According to Baker and Cook (1974) and Boyetchko (1999) biological control is "decreasing the density of inoculums or disease fabricating actions of pathogen or parasite in its dynamic or static state, by one or more organisms, accomplished naturally or through alteration of surroundings, host or antagonist ".

Entomopathogenic fungi are potential biological control agents with a wide host range comprising over 100 genera with approximately 750 species (Hasan, 2014). Out of 31 insect orders, 20 are infected by entomopathogenic fungi in all the developmental stages (Araujo and Hughes, 
2016). L. lecanii is one of several Deuteromycetes species and a potential biocontrol agent of insect order Homoptera, most commonly aphids, scale insects and whiteflies in tropical and subtropical regions. Infected insects develop white mycelial growth all over the body, hence the fungus is commonly called as "white-halo" fungus. The effectiveness of $L$. lecanii was studied and demonstrated first in India by Easwaramoorthi and Jayaraj (1978). Temperature and relative humidity are the major environmental factors, which affect the epizootics of L. lecanii under field conditions (Shinde et al., 2010). Entomopathogenic fungi perform well under optimum temperature $\left(25 \pm 1^{\circ} \mathrm{C}\right)$ and high relative humidity $(>70 \%)$. Extreme temperatures and poor relative humidity limits the use of these entompathogens in rabi and summer seasons and arid climate. To overcome this, there is a need to develop a suitable formulation for the successful utilization of mycoinsecticides. A good formulation helps in preserving organisms, delivering them to their target insect and to improve their activities.

Biological and physical properties of the formulation must remain stable for at least one year, but preferably for more than 18 months for commercialization to take place (Couch and Ignoffo, 1981). Keeping this in view the following study was carried out to evaluate wettable powder and oil based formulations of Lecanicillium lecanii (Zimmermann) $\mathrm{IOF}_{1}$ strain (KM215209) under invitro conditions.

\section{Materials and Methods}

A laboratory experiment was carried out to prepare and evaluate the wettable powder formulation and different combinations of oil based formulations of $L$. lecanii at the Institute of Organic Farming (IOF), University of Agricultural Sciences, Dharwad.

\section{Isolation and maintenance of pure cultures of L. lecanii}

The pure culture of $L$. lecanii was isolated from infected spiralling whiteflies collected from the guava orchard. The infected whiteflies have white mycelial growth on the surface of the body. The mycelial growth was taken with the help of inoculation loop, the inoculums was transferred in to a sterile culture petri plates containing SMAY media. The plates were incubated at room temperature $26 \pm 1{ }^{\circ} \mathrm{C}$ at $80 \% \mathrm{RH}$ for three days and the colonies that came up were further purified by repeated subculture on SMAY media. The isolates that came up on the SMAY medium were identified as $L$. lecanii by microscopic examination according to the outlines given by Samson et al., (1988) and maintained as pure culture.

\section{Mass production procedure for $L$. lecanii and M. anisopliae}

Mass production procedure for L. lecanii and M. anisopliae is similar but only the culture is different as per method developed by Lingappa and Patil (2002).

\section{Flow chart for mass production of entomopathogens}

Broken rice ( $250 \mathrm{~g}$ ) was taken in $1 \mathrm{~kg}$ capacity polypropanyle bag Added $250 \mathrm{ml}$ of $1 \%$ yeast extract solution prepared in distilled water<smiles>C1CC2CCC(C1)CC2</smiles>

Soaked overnight

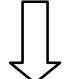


Sterilized under autoclave at 15 PSI for $30 \mathrm{~min}$<smiles>C1CCC1</smiles>

After cooling to room temperature inoculated with $2 \mathrm{ml}$ suspension $\left(10^{6}\right.$ conidia/ml $)$ under laminar air flow

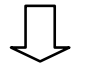

Incubated at room temperature $\left(26 \pm 1^{\circ} \mathrm{C}\right)$ condition for 20 days at high $\mathrm{RH}(>80 \%)$ harvested and air dried digested material<smiles>C1CCC1</smiles>

Ground the digested material and dried once again to bring down moisture to below $8 \%$<smiles>C1CCC1</smiles>

Then sieved the digested material through 344 sieve meshes in order to get pure spore for further preparation of different formulations.

\section{Preparation of oil based formulation}

The oil based formulation of L. lecanii were prepared by using freshly harvested four grams of L. lecanii dry conidia $\left(10^{9}\right.$ spores/ $\left.\mathrm{g}\right)$ obtained from broken rice for which $20 \mathrm{ml}$ of oils $+20 \mathrm{ml}$ glycerol, were mixed and homogenized by using vertical mixture for five minutes for proper encapsulation of spores and required quantity of distilled water was added $+0.1 \%$ of tween- 80 as spreading agent of spores.

Then stored both under ambient temperature and refrigerated conditions in a plastic container (50 ml capacity) for further study (Table 1). The different combination of oil based formulations of $L$. lecanii are as detailed below.

1) Rice bran oil formulation: $4 \mathrm{~g}$ of dry conidia $\left(10^{9}\right.$ spores/g) $+20 \mathrm{ml}$ Rice bran oil $+20 \mathrm{ml}$ glycerol $+956 \mathrm{ml}$ distilled water + $0.1 \%$ tween 80 .

2) Olive oil formulation: $4 \mathrm{~g}$ of dry conidia $\left(10^{9}\right.$ spores/g) $+20 \mathrm{ml}$ olive oil +20 $\mathrm{ml}$ glycerol $+956 \mathrm{ml}$ distilled water $+0.1 \%$ tween 80 .
3) Rice bran $(60 \%)+$ Corn oil $(40 \%)$ formulation: $4 \mathrm{~g}$ of dry conidia $\left(10^{9}\right.$ spores/ g) $+20 \mathrm{ml}$ Rice bran + corn oil $+20 \mathrm{ml}$ glycerol $+956 \mathrm{ml}$ distilled water $+0.1 \%$ tween 80 .

\section{Preparation of wettable powder formulation}

Ten grams of dried conidia of L. lecanii cultured on broken rice grains $\left(10^{9} \mathrm{cfu} / \mathrm{g}\right)$ mixed with $90 \mathrm{~g}$ of carrier material (talc) to get formulated $10^{8} \mathrm{cfu} / \mathrm{g}$ of product.

Before mixing the carrier material sieved through 355 mesh size sieves to maintain uniformity in particle size of conidial powder.

The carrier material sterilized in an autoclave at $121^{\circ} \mathrm{C}$ and 15 Psi for $30 \mathrm{~min}$ and mixed with conidial powder after two days. After that $50 \mathrm{~g}$ of this formulation was packed in small polyethylene bags.

One set of bags stored in ambient room temperature $\left(26+1^{\circ} \mathrm{C}\right.$ ART $)$ and another set under refrigerated $\left(4^{0} \mathrm{C} ; \mathrm{RC}\right)$ condition. 


\section{Spore assessment}

One gram of fungal spores developed on broken rice and sieved under 344 mesh were taken and diluted with $9 \mathrm{ml}$ of sterile distilled water. To the 1-2 drops of Tween-80 was added for uniform distribution of spores in the water. Then the suspension was serially diluted up to dilution of $10^{-6}$ and $10^{-7}$. From which $1 \mathrm{ml}$ of suspension was drawn and the number of conidia per ml were determined by using Neubaeur's haemocytometer under phase contrast microscope (Plate 2).

The number of spores / $g$ was calculated by using the following formula

Number of spores $/ \mathrm{g}=$

Number of spores Present

Number of cells $-\mathrm{X} 400 \times 0.1 \times 1000 \times \mathrm{DF}$

Where, DF: Dilution factor, 0.1: Depth factor, 1000: Conversion factor

Efficacy of oil based formulations of $L$. lecanii against sucking insect pests under laboratory conditions

Different sucking pests viz., corn aphid, cotton thrips, mealybug, spiralling whitefly and soybean mite were used for assessment of bio efficacy of different oil based formulations and wettable powder formulation of $L$. lecanii under laboratory condition.

The field collected sucking pest's viz.,corn aphids, cotton thrips, mealybugs, spiralling whitefly and soybean mites are maintained in field cage containing host plants (maize for aphid, soybean for mite, cotton for thrips, pumpkin for mealybug and flemingia for spiralling whitefly) for multiplication. After multiplication of these pests, the uniform sized aphid, thrips, mealybugs, spiralling whitefly and mites were released in petriplate containing different host leaves placed on water soaked blotting paper and each treatment was replicated three times in each replication 25 aphids were released, similarly in case of cotton thrips, mealybugs, soybean mites and whiteflies 25 individuals were placed in each petriplate for each replicated thrice. After that different concentration of oil based formulations $(1.00 \mathrm{ml}, 1.50 \mathrm{ml}, 2.00$ $\mathrm{ml}, 2.50 \mathrm{ml}$ and $3.00 \mathrm{ml}$ of stock solution containing $10^{6} \mathrm{cfu} / \mathrm{ml}$ added to 1 litre of water and wettable powder formulation (1.00 $\mathrm{g}, 1.50 \mathrm{~g}, 2.00 \mathrm{~g}, 2.50 \mathrm{~g}$ and $3.00 \mathrm{~g} /$ litre of water) form that $1 \mathrm{ml}$ of spray solution was sprayed on the test insect by using potter spray tower $(15 \mathrm{lbs}$ per square $\mathrm{cm}$ ) to get uniform distribution of conidia on test insects and kept them in the environmental chamber (26 $\pm 1^{\circ} \mathrm{C}$ temperature and $80 \pm 5 \% \mathrm{RH}$ ) for sporulation. For the control distilled water spray was used, the mortality of test insects was recorded daily $\left(1,2,3,4\right.$, and $5^{\text {th }}$ day) till the death of all test insects. The data on per cent corrected mortality was finding out by using Abbots formula.

Per cent corrected mortality $=$

Y Number of grubs dead in control $X$ Number of grubs dead in treatment $-\mathrm{X} 100$

$\mathrm{X}$ Total number of grubs used in control Number of grubs dead in control

\section{Results and Discussion}

The different $L$. lecanii oil based formulations such as rice bran oil, rice bran $(60 \%)+$ corn oil $(40 \%)$ and olive oil formulations were evaluated against sucking pests under in vitro conditions (Table 2-7). The results of the present findings revealed that the all the sucking pests viz., corn aphid, grapevine mealybug, cotton thrips and spiralling 
whitefly showed more susceptibility to the oil based formulation, rice bran oil $(60 \%)+$ corn oil $(40 \%)$ which recorded lower $\mathrm{LC}_{50}$ value to the corn aphid $\left(0.182 \times 10^{6} \mathrm{cfu} / \mathrm{ml}\right)$, grapevine mealybug $\left(0.560 \times 10^{6} \mathrm{cfu} / \mathrm{ml}\right)$, cotton thrips $\left(0.591 \times 10^{6} \mathrm{cfu} / \mathrm{ml}\right)$ and guava whitefly $\left(0.942 \times 10^{6} \mathrm{cfu} / \mathrm{ml}\right)$ which was followed by other two oil based formulations such as olive oil and rice bran oil formulation. However, the olive oil based formulation was found best to soybean mite recorded least $\mathrm{LC}_{50}$ value $0.674 \times 10^{6} \mathrm{cfu} / \mathrm{ml}$. The wettable powder formulation recorded highest $\mathrm{LC}_{50}$ value against corn aphid $\left(0.261 \times 10^{8} \mathrm{cfu} / \mathrm{g}\right)$, grapevine mealy bug $\left(0.740 \times 10^{8} \mathrm{cfu} / \mathrm{g}\right)$, cotton thrips $\left(1.019 \times 10^{8} \mathrm{cfu} / \mathrm{g}\right)$, guava whitefly $\left(1.757 \times 10^{8} \mathrm{cfu} / \mathrm{g}\right)$ and soy bean mite $\left(0.917 \times 10^{8} \mathrm{cfu} / \mathrm{g}\right)$ at $120 \mathrm{~h}$.

The present finding regarding the superiority of oil based formulation of L. lecanii are in agreement with the findings of Kim et al., (2001) who demonstrated that L. lecanii (VL10 isolate) oil based formulation was highly pathogenic against Myzus persicae. Similar results reported by Yokomi and Gottwald, 1988, observed LC $_{50}$ value of 1.65 $\times 10^{6} \mathrm{cfu} / \mathrm{ml}$ against Myzus persicae. Asi et al., (2009) also reported that the fungal isolate Verticillium lecanii (V17) with $\mathrm{LC}_{50}$ of $1.88 \times$ $10^{6} \mathrm{cfu} / \mathrm{ml}$ was considered the most effective against the aphids. Similarly, Sarnaya et al., (2010), recorded that the lowest $\mathrm{LC}_{50}$ value of L. lecanii isolate against cowpea aphid, $A$. craccivora $\left(2.5 \times 10^{4} \mathrm{cfu} / \mathrm{ml}\right), B$. brassicae $\left(1.2 \times 10^{4} \mathrm{cfu} / \mathrm{ml}\right)$, A. gossypii $\left(2.7 \times 10^{4} \mathrm{cfu}\right.$ $/ \mathrm{ml})$.

According to Halyer (1993) who reported that addition of rape seed oil to the fungus $V$. lecanii at $1 \times 10^{8} \mathrm{cfu} / \mathrm{ml}$ increased efficacy up to 90 per cent when tested on aphid, Aphis gossypii (Glover) and thrips, Frankliniella occidentalis (Pergande), and also in comparison with Ramarethinam et al.,(2000) who reported that the Bio power, a commercial formulation of $V$. lecanii cause 43.56 per cent mortality on thrips, Scirtothrips dorsalis (Hood) on chilli.

The present findings are in line with Harischandra and Shekharappa (2008) reported that the oil based formulation of $V$. lecanii at $1 \times 10^{8} \mathrm{cfu} / \mathrm{ml}$, observed 98 per cent mortality of okra aphid at $10^{\text {th }}$ day after treatment followed by wettable powder formulation (96.67\%). Similarly, Mote et al., (2003) reported that higher mortality of gerbera aphid was observed in oil based formulation of $V$. lecanii at $0.3 \%(93.44 \%)$ than wettable powder formulation $(91.67 \%)$.

Table.1 Treatment details of different entomopathogenic fungi formulations of L. lecanii IOF1 strain (KM215209)

\begin{tabular}{|l|l|l|l|l|l|}
\hline \multicolumn{1}{|c|}{ Treatments } & \multicolumn{5}{|c|}{ Dosage ( $\mathrm{g}$ or ml/ lit of water) } \\
\hline \multicolumn{4}{|c|}{ Oil based and wettable powder formulations of $\mathrm{L}$. lecanii } \\
\hline$T_{1}$ - Rice bran oil formulation $\left(10^{6} \mathrm{cfu} / \mathrm{ml}\right)$ & 1.00 & 1.50 & 2.00 & 2.50 & 3.00 \\
\hline $\begin{array}{l}T_{2}-\text { Rice bran }(60 \%)+\text { corn oil }(40 \%) \\
\text { formulation }\left(10^{6} \mathrm{cfu} / \mathrm{ml}\right)\end{array}$ & 1.00 & 1.50 & 2.00 & 2.50 & 3.00 \\
\hline$T_{3}-$ Olive oil formulation $\left(10^{6} \mathrm{cfu} / \mathrm{ml}\right)$ & 1.00 & 1.50 & 2.00 & 2.50 & 3.00 \\
\hline$T_{4}-$ Wettable powder formulation $\left(10^{8} \mathrm{cfu} / \mathrm{g}\right)$ & 1.00 & 1.50 & 2.00 & 2.50 & 3.00 \\
\hline$T_{5}-$ Control & \multicolumn{5}{c|}{ Distilled water spray } \\
\hline
\end{tabular}


Table.2 Median lethal concentration (LC50) of oil based formulations of L. lecanii IOF1 strain (KM215209) against corn aphid, Rhopalsiphum maidis (Fitch)

\begin{tabular}{|c|c|c|c|c|c|c|}
\hline \multirow[t]{2}{*}{ Formulation } & \multirow[t]{2}{*}{$\mathrm{LC}_{50}(\mathrm{cfu} / \mathrm{ml})$} & \multicolumn{2}{|c|}{ Fiducial limits of $\mathrm{LC}_{50}(\mathrm{cfu} / \mathrm{ml})$} & \multirow{2}{*}{$\begin{array}{c}\text { Regression } \\
\text { equation }(Y=a+b x)\end{array}$} & \multirow[t]{2}{*}{$\mathrm{LC}_{95}(\mathrm{cfu} / \mathrm{ml})$} & \multirow[t]{2}{*}{$\chi^{2}$} \\
\hline & & Lower limit & Upper limit & & & \\
\hline $\begin{array}{l}\text { Rice bran oil } \\
(60 \%)+\text { corn oil } \\
(40 \%) \\
\text { formulation }\end{array}$ & $0.182 \times 10^{6}(\mathrm{cfu} / \mathrm{ml})$ & $0.044 \times 10^{6}(\mathrm{cfu} / \mathrm{ml})$ & $0.347 \times 10^{6}(\mathrm{cfu} / \mathrm{ml})$ & $Y=1.015+0.082 x$ & $2.883 \times 10^{6}(\mathrm{cfu} / \mathrm{ml})$ & 0.379 \\
\hline $\begin{array}{l}\text { Olive oil } \\
\text { formulation }\end{array}$ & $0.266 \times 10^{6}(\mathrm{cfu} / \mathrm{ml})$ & $0.077 \times 10^{6}(\mathrm{cfu} / \mathrm{ml})$ & $0.461 \times 10^{6}(\mathrm{cfu} / \mathrm{ml})$ & $Y=0.700+0.074 x$ & $5.981 \times 10^{6}(\mathrm{cfu} / \mathrm{ml})$ & 1.689 \\
\hline $\begin{array}{l}\text { Rice bran oil } \\
\text { formulation }\end{array}$ & $0.316 \times 10^{6}(\mathrm{cfu} / \mathrm{ml})$ & $0.147 \times 10^{6}(\mathrm{cfu} / \mathrm{ml})$ & $0.481 \times 10^{6}(\mathrm{cfu} / \mathrm{ml})$ & $Y=0.769+0.074 x$ & $6.114 \times 10^{6}(\mathrm{cfu} / \mathrm{ml})$ & 0.582 \\
\hline $\begin{array}{l}\text { Wettable powder } \\
\text { formulation }\end{array}$ & $0.261 \times 10^{8}(\mathrm{cfu} / \mathrm{g})$ & $0.060 \times 10^{8}(\mathrm{cfu} / \mathrm{g})$ & $0.475 \times 10^{8}(\mathrm{cfu} / \mathrm{g})$ & $Y=0.718+0.083 x$ & $5.674 \times 10^{8}(\mathrm{cfu} / \mathrm{g})$ & 0.523 \\
\hline
\end{tabular}

Table.3 Median lethal concentration (LC50) of oil based formulations of L. lecanii IOF1 strain (KM215209) against grape vine mealybug, Maconellicoccus hirsutus (Green)

\begin{tabular}{|c|c|c|c|c|c|c|}
\hline \multirow[t]{2}{*}{ Formulation } & \multirow[t]{2}{*}{$\mathrm{LC}_{50}(\mathrm{cfu} / \mathrm{ml})$} & \multicolumn{2}{|c|}{ Fiducial limits of $\mathrm{LC}_{50}(\mathrm{cfu} / \mathrm{ml})$} & \multirow{2}{*}{$\begin{array}{c}\text { Regression } \\
\text { equation }(Y=a+b x)\end{array}$} & \multirow[t]{2}{*}{$\mathrm{LC}_{95}(\mathrm{cfu} / \mathrm{ml})$} & \multirow[t]{2}{*}{$\chi^{2}$} \\
\hline & & Lower limit & Upper limit & & & \\
\hline $\begin{array}{l}\text { Rice bran oil (60 } \\
\%)+ \text { corn oil } \\
(40 \%) \\
\text { formulation }\end{array}$ & $0.560 \times 10^{6}(\mathrm{cfu} / \mathrm{ml})$ & $0.073 \times 10^{6}(\mathrm{cfu} / \mathrm{ml})$ & $1.034 \times 10^{6}(\mathrm{cfu} / \mathrm{ml})$ & $Y=0.361+0.203 x$ & $7.845 \times 10^{6}(\mathrm{cfu} / \mathrm{ml})$ & 0.360 \\
\hline $\begin{array}{l}\text { Olive oil } \\
\text { formulation }\end{array}$ & $0.903 \times 10^{6}(\mathrm{cfu} / \mathrm{ml})$ & $0.024 \times 10^{6}(\mathrm{cfu} / \mathrm{ml})$ & $1.376 \times 10^{6}(\mathrm{cfu} / \mathrm{ml})$ & $Y=0.062+0.195 x$ & $9.401 \times 10^{6}(\mathrm{cfu} / \mathrm{ml})$ & 0.095 \\
\hline $\begin{array}{l}\text { Rice bran oil } \\
\text { formulation }\end{array}$ & $1.287 \times 10^{6}(\mathrm{cfu} / \mathrm{ml})$ & $0.764 \times 10^{6}(\mathrm{cfu} / \mathrm{ml})$ & $1.608 \times 10^{6}(\mathrm{cfu} / \mathrm{ml})$ & $Y=0.249+0.196 x$ & $13.827 \times 10^{6}(\mathrm{cfu} / \mathrm{ml})$ & 2.486 \\
\hline $\begin{array}{l}\text { Wettable powder } \\
\text { formulation }\end{array}$ & $0.740 \times 10^{8}(\mathrm{cfu} / \mathrm{g})$ & $0.131 \times 10^{8}(\mathrm{cfu} / \mathrm{g})$ & $1.207 \times 10^{8}(\mathrm{cfu} / \mathrm{g})$ & $Y=0.189+0.198 x$ & $10.206 \times 10^{8}(\mathrm{cfu} / \mathrm{g})$ & 0.219 \\
\hline
\end{tabular}


Table.4 Median lethal concentration (LC50) of oil based formulations of L. lecanii IOF1 strain (KM215209) against cotton thrips, Thrips tabaci (Linde)

\begin{tabular}{|c|c|c|c|c|c|c|}
\hline \multirow[t]{2}{*}{ Formulation } & \multirow[t]{2}{*}{$\mathrm{LC}_{50}(\mathrm{cfu} / \mathrm{ml})$} & \multicolumn{2}{|c|}{ Fiducial limits of $\mathrm{LC}_{50}(\mathrm{cfu} / \mathrm{ml})$} & \multirow{2}{*}{$\begin{array}{c}\text { Regression } \\
\text { equation }(Y=a+b x)\end{array}$} & \multirow[t]{2}{*}{$\mathrm{LC}_{95}(\mathrm{cfu} / \mathrm{ml})$} & \multirow[t]{2}{*}{$\chi^{2}$} \\
\hline & & Lower limit & Upper limit & & & \\
\hline $\begin{array}{l}\text { Rice bran oil }(60 \%)+ \\
\text { corn oil }(40 \%) \\
\text { formulation }\end{array}$ & $0.591 \times 10^{6}(\mathrm{cfu} / \mathrm{ml})$ & $0.129 \times 10^{6}(\mathrm{cfu} / \mathrm{ml})$ & $0.921 \times 10^{6}(\mathrm{cfu} / \mathrm{ml})$ & $Y=0.457+0.179 x$ & $3.924 \times 10^{6}(\mathrm{cfu} / \mathrm{ml})$ & 0.486 \\
\hline Olive oil formulation & $0.751 \times 10^{6}(\mathrm{cfu} / \mathrm{ml})$ & $0.188 \times 10^{6}(\mathrm{cfu} / \mathrm{ml})$ & $1.098 \times 10^{6}(\mathrm{cfu} / \mathrm{ml})$ & $Y=0.239+0.182 x$ & $5.378 \times 10^{6}(\mathrm{cfu} / \mathrm{ml})$ & 1.176 \\
\hline $\begin{array}{l}\text { Rice bran oil } \\
\text { formulation }\end{array}$ & $1.068 \times 10^{6}(\mathrm{cfu} / \mathrm{ml})$ & $0.686 \times 10^{6}(\mathrm{cfu} / \mathrm{ml})$ & $1.313 \times 10^{6}(\mathrm{cfu} / \mathrm{ml})$ & $Y=0.077+0.176 x$ & $4.361 \times 10^{6}(\mathrm{cfu} / \mathrm{ml})$ & 1.684 \\
\hline $\begin{array}{l}\text { Wettable powder } \\
\text { formulation }\end{array}$ & $1.019 \times 10^{8}(\mathrm{cfu} / \mathrm{g})$ & $0.409 \times 10^{8}(\mathrm{cfu} / \mathrm{g})$ & $1.355 \times 10^{8}(\mathrm{cfu} / \mathrm{g})$ & $Y=0.017+0.197 x$ & $6.238 \times 10^{8}(\mathrm{cfu} / \mathrm{g})$ & 0.685 \\
\hline
\end{tabular}

Table.5 Median lethal concentration (LC50) of oil based formulations of L. lecanii IOF1 strain (KM215209) against spiralling whitefly, Trialeurodes vaporariorum (Westwood)

\begin{tabular}{|c|c|c|c|c|c|c|}
\hline \multirow[t]{2}{*}{ Formulation } & \multirow[t]{2}{*}{$\mathrm{LC}_{50}(\mathrm{cfu} / \mathrm{ml})$} & \multicolumn{2}{|c|}{ Fiducial limits of $\mathrm{LC}_{50}(\mathrm{cfu} / \mathrm{ml})$} & \multirow{2}{*}{$\begin{array}{c}\text { Regression } \\
\text { equation }(Y=a+b x)\end{array}$} & \multirow[t]{2}{*}{$\mathrm{LC}_{95}(\mathrm{cfu} / \mathrm{ml})$} & \multirow[t]{2}{*}{$\chi^{2}$} \\
\hline & & Lower limit & Upper limit & & & \\
\hline $\begin{array}{l}\text { Rice bran oil }(60 \%) \\
+ \text { corn oil }(40 \%) \\
\text { formulation }\end{array}$ & $0.942 \times 10^{6}(\mathrm{cfu} / \mathrm{ml})$ & $0.517 \times 10^{6}(\mathrm{cfu} / \mathrm{ml})$ & $1.213 \times 10^{6}(\mathrm{cfu} / \mathrm{ml})$ & $Y=0.067+0.183 x$ & $4.137 \times 10^{6}(\mathrm{cfu} / \mathrm{ml})$ & 1.101 \\
\hline Olive oil formulation & $1.283 \times 10^{6}(\mathrm{cfu} / \mathrm{ml})$ & $0.840 \times 10^{6}(\mathrm{cfu} / \mathrm{ml})$ & $1.571 \times 10^{6}(\mathrm{cfu} / \mathrm{ml})$ & $Y=0.221+0.158 x$ & $7.204 \times 10^{6}(\mathrm{cfu} / \mathrm{ml})$ & 1.195 \\
\hline $\begin{array}{l}\text { Rice bran oil } \\
\text { formulation }\end{array}$ & $1.530 \times 10^{6}(\mathrm{cfu} / \mathrm{ml})$ & $1.209 \times 10^{6}(\mathrm{cfu} / \mathrm{ml})$ & $1.788 \times 10^{6}(\mathrm{cfu} / \mathrm{ml})$ & $Y=0.483+0.173 x$ & $7.516 \times 10^{6}(\mathrm{cfu} / \mathrm{ml})$ & 1.754 \\
\hline $\begin{array}{l}\text { Wettable powder } \\
\text { formulation }\end{array}$ & $1.757 \times 10^{8}(\mathrm{cfu} / \mathrm{g})$ & $1.464 \times 10^{8}(\mathrm{cfu} / \mathrm{g})$ & $2.052 \times 10^{8}(\mathrm{cfu} / \mathrm{g})$ & $Y=0.651+0.176 x$ & $7.299 \times 10^{8}(\mathrm{cfu} / \mathrm{g})$ & 5.075 \\
\hline
\end{tabular}


Table.6 Median lethal concentration (LC50) of oil based formulations of L. lecanii IOF1 strain (KM215209) against soybean mite, Tetranychus urticae (Koch)

\begin{tabular}{|c|c|c|c|c|c|c|}
\hline \multirow[t]{2}{*}{ Formulation } & \multirow[t]{2}{*}{$\mathrm{LC}_{50}(\mathrm{cfu} / \mathrm{ml})$} & \multicolumn{2}{|c|}{ Fiducial limits of $\mathrm{LC}_{50}(\mathrm{cfu} / \mathrm{ml})$} & \multirow{2}{*}{$\begin{array}{c}\text { Regression } \\
\text { equation }(Y=a+b x)\end{array}$} & \multirow[t]{2}{*}{$\mathrm{LC}_{95}(\mathrm{cfu} / \mathrm{ml})$} & \multirow[t]{2}{*}{$\chi^{2}$} \\
\hline & & Lower limit & Upper limit & & & \\
\hline Olive oil formulation & $0.674 \times 10^{6}(\mathrm{cfu} / \mathrm{ml})$ & $0.210 \times 10^{6}(\mathrm{cfu} / \mathrm{ml})$ & $1.174 \times 10^{6}(\mathrm{cfu} / \mathrm{ml})$ & $Y=0.220+0.192 x$ & $4.746 \times 10^{6}(\mathrm{cfu} / \mathrm{ml})$ & 0.378 \\
\hline $\begin{array}{l}\text { Rice bran oil } \\
\text { formulation }\end{array}$ & $0.744 \times 10^{6}(\mathrm{cfu} / \mathrm{ml})$ & $0.036 \times 10^{6}(\mathrm{cfu} / \mathrm{ml})$ & $1.172 \times 10^{6}(\mathrm{cfu} / \mathrm{ml})$ & $Y=0.210+0.200 x$ & $6.542 \times 10^{6}(\mathrm{cfu} / \mathrm{ml})$ & 0.708 \\
\hline $\begin{array}{l}\text { Rice bran oil }(60 \%) \\
+ \text { corn oil }(40 \%) \\
\text { formulation }\end{array}$ & $0.901 \times 10^{6}(\mathrm{cfu} / \mathrm{ml})$ & $0.409 \times 10^{6}(\mathrm{cfu} / \mathrm{ml})$ & $1.207 \times 10^{6}(\mathrm{cfu} / \mathrm{ml})$ & $Y=0.096+0.173 x$ & $7.377 \times 10^{6}(\mathrm{cfu} / \mathrm{ml})$ & 0.875 \\
\hline $\begin{array}{l}\text { Wettable powder } \\
\text { formulation }\end{array}$ & $0.917 \times 10^{8}(\mathrm{cfu} / \mathrm{g})$ & $0.080 \times 10^{8}(\mathrm{cfu} / \mathrm{g})$ & $1.342 \times 10^{8}(\mathrm{cfu} / \mathrm{g})$ & $Y=0.059+0.193 x$ & $9.194 \times 10^{8}(\mathrm{cfu} / \mathrm{g})$ & 0.354 \\
\hline
\end{tabular}

Table.7 Comparisons of median lethal concentration (LC50) different oil based formulations of L. lecanii IOF1 strains (KM215209) against different sucking pests

\begin{tabular}{|l|c|c|c|c|c|}
\hline \multicolumn{1}{|c|}{ Formulations } & \multicolumn{1}{|c|}{ Corn aphid } & $\begin{array}{c}\text { Grape vine } \\
\text { mealybug }\end{array}$ & Cotton thrips & Gauva whitefly & Soybean mite \\
\hline $\begin{array}{l}\text { Rice bran oil }(\mathbf{6 0} \%) \\
+ \\
\text { forn oil } \mathbf{4 0 \%})\end{array}$ & $0.182 \times 10^{6}(\mathrm{cfu} / \mathrm{ml})$ & $0.560 \times 10^{6}(\mathrm{cfu} / \mathrm{ml})$ & $0.591 \times 10^{6}(\mathrm{cfu} / \mathrm{ml})$ & $0.942 \times 10^{6}(\mathrm{cfu} / \mathrm{ml})$ & $0.901 \times 10^{6}(\mathrm{cfu} / \mathrm{ml})$ \\
\hline $\begin{array}{l}\text { Olive oil } \\
\text { formulation }\end{array}$ & $0.266 \times 10^{6}(\mathrm{cfu} / \mathrm{ml})$ & $0.903 \times 10^{6}(\mathrm{cfu} / \mathrm{ml})$ & $0.751 \times 10^{6}(\mathrm{cfu} / \mathrm{ml})$ & $1.283 \times 10^{6}(\mathrm{cfu} / \mathrm{ml})$ & $0.674 \times 10^{6}(\mathrm{cfu} / \mathrm{ml})$ \\
\hline $\begin{array}{l}\text { Rice bran oil } \\
\text { formulation }\end{array}$ & $0.316 \times 10^{6}(\mathrm{cfu} / \mathrm{ml})$ & $1.287 \times 10^{6}(\mathrm{cfu} / \mathrm{ml})$ & $1.068 \times 10^{6}(\mathrm{cfu} / \mathrm{ml})$ & $1.530 \times 10^{6}(\mathrm{cfu} / \mathrm{ml})$ & $0.744 \times 10^{6}(\mathrm{cfu} / \mathrm{ml})$ \\
\hline $\begin{array}{l}\text { Wettable powder } \\
\text { formulation }\end{array}$ & $0.261 \times 10^{8}(\mathrm{cfu} / \mathrm{g})$ & $0.740 \times 10^{8}(\mathrm{cfu} / \mathrm{g})$ & $1.019 \times 10^{8}(\mathrm{cfu} / \mathrm{g})$ & $1.757 \times 10^{8}(\mathrm{cfu} / \mathrm{g})$ & $0.917 \times 10^{8}(\mathrm{cfu} / \mathrm{g})$ \\
\hline
\end{tabular}


In the present study, the superiority of oil based formulation of $L$. lecanii to the cotton thrips were more susceptible to oil based formulation which shows the early morality to the oil based formulation. These findings are conformity with the results of Mote et al., (2003) who reported that the oil based formulation of V.lecanii @0.3\% recorded more than 91.67 per cent mortality of Gerbera thrips in polyhouse at 14 days after treatment compared to wettable powder (WP) @ 0.3\% which causes less than 88.33 per cent mortality.

The efficacy results of three oil based formulations of $L$. lecanii against soybean mite, $T$. urticae revealed that the olive oil based formulation with least $\mathrm{LC}_{50}$ value $\left(0.674 \times 10^{6} \mathrm{cfu} / \mathrm{ml}\right)$ compared to other oil based formulations which proved to be the best used for mite control. These findings corroborated with the report of Amjad et al., (2012) who reported that the oil based formulation of $V$. lecanii (V17) isolate recorded lower $\operatorname{LC}_{50}\left(5.7 \times 10^{6} \mathrm{cfu} / \mathrm{ml}\right)$ after inoculation which showed the most virulent strain against mite, $T$. urticae. The $V$. lecanii at $0.3 \%$ of oil based formulation recorded 82.40 per cent mortality of Tetranychus urticae infesting gerbera at $14^{\text {th }}$ day after treatment in green house (Mote et al., 2003).

According to Harischandra and Shekharappa (2008) reported that the oil based formulation of $V$. lecanii $1 \times 10^{8} \mathrm{cfu} / \mathrm{ml}$ recorded the highest per cent mortality $(97.00 \%)$ against okra thrips, followed by wettable powder formulation at $10^{\text {th }}$ day after spray. The present study also in agreement with earlier report of Nier et al., (1993) who reported that pathogencity of $V$. lecanii against spiralling whitefly, T. vaporariorum and Bemisia tabaci (Gennadius), at the concentration of $3.2 \times 10^{6}$ cfu/ $\mathrm{ml}$ resulting in 92 and 100 percent mortality, respectively after 7 days after treatment. The results of the present investigation indicated more virulence of oil based formulation found more effective at lower concentration compared to wettable powder formulation, It is due to the oil based formulation prevented the desiccation of the conidia and helps in longer survival period and better penetration of peg into the integuments as per the report of (Burges, 1998).

From the present study it is evident that oil based formulations of entomopathogenic fungi are more effective than wettable powder formulation under laboratory condition. This efficacy can be attributed to oil based formulations which prevented that spores from desiccation and increased viability. Oil formulations are compatible with other integrated pest management approaches. These formulations provide scope for the application of entomopathogens in arid climate where the temperature and relative humidity are major constraints.

\section{References}

Amjad, M., Bashir, M. H., Afzal, M., Sabri, M. A. and Javed, N., 2012, Synergistic effect of some entomopathogenic fungi and synthetic pesticides, against two spotted spider mite, Tetranychus urticae Koch (Acari: Tetranychidae). Pak. J. Zool., 44 (4): 977-984.

Araujo, J. and Hughes, D.P., 2016, Diversity of Entomopathogenic Fungi: Which Groups Conquered the Insect Body? Adv. Genet., 94, 1 - 39.

Asi, M. R., Bashir, M. H., Afzal, M. and Imran, S., 2009, Effect of conidial concentration of entomopathogenic fungi on mortality of cabbage aphid, Brevicoryne brassicae L. Pak. J. Life Soc. Sci., (2): 175-180.

Boyetchko, S.M., 1999, Biotechnological approaches in biocontrol of plant pathogens. In: Mukerji, K.G., Chamola, B.P., Upadhyay, R.K. (Eds.), Springer Science+Business Media, New York, pp. 
$51-71$.

Baker, K. F. and Cook, J., 1974, Biological control of plant pathogens. Cambridge University Press. USA.

Burges, H. D., 1998, Formulation of Microbial Biopesticides, Kluwer Academic Publishers, London, UK, p. 412.

Digvijay Singh, Tanveer Kour Raina and Joginder Singh, 2017, Entomopathogenic fungi: An effective biocontrol agent for management of insect populations naturally. J. Pharm. Sci. \& Res., 9(6):830839.

Easwaramoorthy, S. and Jayaraj, S., 1978, Effectiveness of the white halo fungus, Cephalosporium lecanii, against field populations of coffee green bug, Coccus viridis. J. Invertebr. Pathol., 32: 88-96.

Halyer, N., 1993, Verticillium lecanii for control of aphids and thrips on cucumber. Bull. OILB/SROP, 16: 63-66.

Harischandra, N. R. and Shekharappa, 2008, In vitro evaluation of entomopathogenic fungal formulations against sucking insect pests of okra. Karnataka J. Agric. Sci., 22 (4): 784-786.

Hasan, S., 2014, Entomopathogenic fungi as potent agents of biological control, Int. J. Eng. Tech. Res., 2(3): 221-229.

Kim, J. J., Lee, M. H., Yoon, C. S., Kim, H. S., Yoo, J. K. and Kim, K. C., 2001, Control of cotton aphid and greenhouse whitefly with a fungal pathogen. In: Biological control of greenhouse pests. Food and fertilizer technology centre extension bulletin 502. Food and fertilizer technology centre, Taipei, Taiwan, pp. 815.

Lingappa, S. and Patil, R. K., 2002, Nomuraea rileyi - A Potential Mycoinsecticide. Univ. Agric. Sci., Dharwad, p. 30.

Mote, U. N., Mahajan, P. R. and Kadam, J. R., 2003, Bioefficacy of Verticillium lecanii (Zimm.) Viegas against sucking pests of gerbera in polyhouse. In: Green Pesticides for Insect Pest Management, Edts. Ignacimuthu S. J. and Jayaraj. S., Narosa publishing house, Chennai, pp. 179-183.

Nier, T., River, F. and Bermudez, J. C., 1993, First report of Mexico on the isolation of Verticillium lecanii from whitefly and in vitro pathogencity tests on this insect. Rev. Mex. De Mycol., 7: 149-156.

Ramarethinam, S., Marimuthu, S., Murugesan, N. V. and Lognathan, S., 2000, Evaluation of Paecilomyces fumosoroseus, on entomopathogenic fungus for controlling red spider mite, Oligonychus coffeae (Nietner) (Acarina: Tetranychidae), infesting tea in India. Pestology, 24 (9): 1-5.

Sarnaya, S., Ushakumari, R., Jacob, S. and Philip, B. M., 2010, Efficacy of different entomopathogenic fungi against cowpea aphid, Aphis craccivora (Koch). $J$. BioPestic., 3(1): 138-142.

Shinde, S. V., Patel, K. G., Purohit, M. S., Pandya, J. R. and Sabalpara, A. N., 2010, Lecanicillium lecanii (Zimm.) Zare and Games" an important biocontrol agent for the management of insect pests - a review. Agri. Review, 31 (4): 235 - 252.

Yokomi, R. K. and Gottwald, T. R., 1988, Virulence of Verticillium lecanii isolated in aphids determined by detached leaf bioassay. J. Invert. Path., 51: 250-258.

\section{How to cite this article:}

Sharanabasappa M. Ganganalli and Patil, R.K. 2019. Development and Evaluation of Median Lethal Concentration (LC50) of Wettable Powder and Oil Based Formulations of Lecanicillium lecanii (Zimmermann) IOF1 Strain (KM215209) under in vitro Conditions. Int.J.Curr.Microbiol.App.Sci. 8(02): 1416-1425. doi: https://doi.org/10.20546/ijcmas.2019.802.165 